PROCEEDINGS OF THE

AMERICAN MATHEMATICAL SOCIETY

Volume 139, Number 6, June 2011, Pages 2087-2092

S 0002-9939(2010)10622-6

Article electronically published on November 9, 2010

\title{
RELATIVELY POINTWISE RECURRENT GRAPH MAP
}

\author{
HATTAB HAWETE
}

(Communicated by Yingfei Yi)

\begin{abstract}
Let $f$ be a self-continuous map of a graph $G$. Let $P(f)$ and $R(f)$ denote the sets of periodic points and recurrent points respectively. We say that the map $f$ is relatively recurrent if $\overline{R(f)}=G$. In this paper, it is shown that $f$ is relatively recurrent if and only if one of the following statements holds:

(a) $G$ is a circle and $f$ is a homeomorphism topologically conjugate to an irrational rotation of the unit circle $\mathbb{S}^{1}$;

(b) $\overline{P(f)}=G$.
\end{abstract}

Part (b) extends a result of Blokh.

\section{INTRODUCTION}

A topological dynamical system is a pair $(X, f)$, where $X$ is a compact metric space and $f$ is a continuous map from $X$ to itself. Let $\mathbb{N}$ be the set of positive integers. Let $f^{0}$ be the identity map of $X$. Define, inductively, $f^{n}=f \circ f^{n-1}$ for any non-zero positive integer $n$. For $x \in X,\left\{f^{n}(x): n \in \mathbb{N}\right\}$ is called the orbit of $x$ and is denoted by $O(x, f)$. Here $x$ is periodic if $f^{n}(x)=x$ for some non-zero positive integer $n$. Also, $x$ is called a recurrent point of $f$ if for any neighborhood $U$ of $x$ and any $m \in \mathbb{N}$ there exists $n>m$ such that $f^{n}(x) \in U$. It is easy to see that if $x$ is recurrent, then every iterate of $x$ is also recurrent. The converse is false. Here $x$ is called an almost periodic point of $f$ if for any neighborhood $U$ of $x$ there exists $N \in \mathbb{N}$ such that $\left\{f^{n+i}(x): i=0,1, \ldots, N\right\} \cap U \neq \emptyset$ for all $n \in \mathbb{N}$. Also, $x$ is a non-wandering point of $f$ provided that for any open set $U$ containing $x$ there exist $y \in U$ and $n \in \mathbb{N}$ such that $f^{n}(y) \in U$. Let $P(f)$, $A P(f), R(f)$ and $\Omega(f)$ denote the set of periodic points, almost periodic points, recurrent points and non-wandering points respectively. Notice that $\Omega(f)$ is closed and for the general topological system $(X, f)$ there are no further relations except for $P(f) \subset A P(f) \subset R(f) \subset \Omega(f)$. But for one-dimensional systems one can say more. For a dendrite map Illanes [8] proved that $\overline{P(f)}=\overline{R(f)}$ if and only if the dendrite does not contain any copy of the Gehman dendrite. For a graph map Mai and Shao [9] showed that $\overline{R(f)}=\overline{P(f)} \cup R(f)$. In Lemma 3.1, for a graph map, we will show that $\overline{A P(f)}=\overline{R(f)}$.

Received by the editors April 10, 2010 and, in revised form, June 2, 2010.

2010 Mathematics Subject Classification. Primary 37B20, 37E25.

Key words and phrases. Periodic point, recurrent point, almost periodic point, minimal set, relatively pointwise recurrent map, graph map.

(C)2010 American Mathematical Society Reverts to public domain 28 years from publication 
It is interesting to study maps such that $P(f)$ or $R(f)$ satisfies some additional properties. Montgomery [12, Weaver [15], Epstein [4], and others studied homeomorphisms such that $P(f)$ is the whole space $X$ ( $X$ is a connected manifold or a continuum embedded in a 2-manifold). For an interval map, Nitecki 13 showed that if $P(f)$ is closed, then $P(f)=\Omega(f)$. For a graph map Mai [1] proved that $R(f)$ is the whole space $X$ if and only if one of the following statements holds:

(1) $X$ is a circle, and $f$ is a homeomorphism topologically conjugate to an irrational rotation of the unit circle;

(2) $f$ is a periodic homeomorphism.

In this paper we will study a relatively pointwise recurrent graph map. Our main result is the following theorem:

Main Theorem. Let $G$ be a graph and let $f: G \rightarrow G$ be a continuous map. Then $f$ is relatively pointwise recurrent if and only if one of the following two statements holds:

(1) $G$ is a circle, and $f$ is a homeomorphism topologically conjugate to an irrational rotation of the unit circle $\mathbb{S}^{1}$;

(2) $\overline{P(f)}=G$.

A set $W \subset X$ is called a minimal set of $f$ if it is nonempty, closed, invariant $(f(W) \subset W)$ and no proper subset of $W$ satisfies these three properties, which is equivalent to the fact that the orbit of every element of $W$ is dense.

In a topological dynamical system there are two well-known theorems which exhibit the close relationship between almost periodic points and minimal sets; see Birkhoff [2]. In fact, if $X$ is a locally compact metric space, then the set of almost periodic points $A P(f)$ is the union of all minimal sets of $f$.

If $X$ has no isolated point, then $f$ is a transitive map if it has a dense orbit. If every orbit of $f$ is dense in $X$, the map $f$ is called minimal. For a transitive graph map Blokh [3] proved that $P(f)$ is dense. In this paper we show that for a graph map if $R(f)$ is dense, then $P(f)$ is also dense, which extends this result of Blokh [3] (see Corollary 3.4).

\section{Relatively POINTWise RECURRENT MAP}

A map $f$ of a compact metric space $(X, d)$ is recurrent if it admits iterates arbitrarily close to the identity, i.e. if there exists a sequence $n_{k} \rightarrow+\infty$ such that

$$
d\left(f^{n_{k}}, I d\right) \rightarrow 0 \quad \text { as } \quad k \rightarrow+\infty .
$$

We say that $f$ is pointwise recurrent if $R(f)=X$.

We continue, motivated by a desire to understand the mechanics of recurrent maps, by a desire to extend some known result and by the following:

In [6] Gottschalk proved that if $X$ is a compact connected metric space, $f$ is a homeomorphism, and if $\overline{R(f)}=X$, then every recurrent cut point of $X$ is periodic.

We start by the following definition.

Definition. $f$ is called a relatively pointwise recurrent map if $\overline{R(f)}=X$.

For example a transitive map is a relatively pointwise recurrent map.

Let $X$ be a closed domain of finite volume of the $n$-Euclidian space $\mathbb{R}^{n}$ or the $n$-torus $\mathbb{T}^{n}$ and let $f$ be an invertible volume-preserving self-map of $X$. Then $f$ is a relatively pointwise recurrent map [7, Theorem 6.1.9]. 
We have the following implications:

recurrent $\Rightarrow^{1}$ pointwise recurrent $\Rightarrow^{2}$ relatively pointwise recurrent.

The following examples show that none of these implications can be reversed.

Examples 2.1. (1) An irrational rotation of $\mathbb{R}^{2}$ is a pointwise recurrent nonrecurrent map.

(2) In $\mathbb{R}^{2}$ we consider the points $A_{n}\left(0, \frac{1}{n}\right)$ and $B\left(n, \frac{1}{n}\right)$ for every integer $n>0$. Put $X=\left(\bigcup_{n>0}\left[A_{n}, B_{n}\right]\right) \cup[0,+\infty[\times\{0\}$. Define the map $f$ on $X$ by:

- $f\left(x, \frac{1}{n}\right)=\left(\varphi(x), \frac{1}{n}\right)$, where

$$
\varphi(x)=\left\{\begin{array}{l}
x+1 \quad \text { if } \quad x \leq n-1, \\
x+1-n \text { if } n-1<x \leq n .
\end{array}\right.
$$

- $f(x, 0)=(x+1,0)$.

$f$ is a relatively pointwise recurrent non-pointwise recurrent map.

In this example one can choose $X$ to be connected.

Theorem 2.2. If a continuous map of a topological space $X$ to itself is either (1) recurrent, (2) pointwise recurrent, or (3) relatively pointwise recurrent, then so is $f^{k}$, for each integer $k$.

Proof. (1) and (2) follow from [14] and [6] respectively.

We have $R(f) \subset R\left(f^{k}\right)$, and so if $\overline{R(f)}=X$, then $\overline{R\left(f^{k}\right)}=X$, which implies (3).

Proposition 2.3. If $f$ is relatively pointwise recurrent, then $f$ is surjective.

Proof. If $y \in R(f)$, then there exists a point $x \in X$ such that $f(x)=y$.

Let $y$ be an element of $X-R(f)$. Then there exists a sequence $y_{n}$ of $R(f)$ which converges to $y$. For all $n$ there exits $x_{n}$ such that $f\left(x_{n}\right)=y_{n}$. Since $X$ is compact, the sequence $x_{n}$ has a limit point $x$ and so $f(x)=y$. Therefore $f$ is surjective.

Proposition 2.4. If $f$ is relatively pointwise recurrent, then $\Omega(f)=X$.

Proof. Let $x$ be an element of $X$. If $x$ is a recurrent point, it is also non-wandering. If $x$ is a non-recurrent point, then every neighborhood of $x$ contains a recurrent point, and so it is in $\Omega(f)$.

The referee noticed that the converse of Proposition 2.4 also holds; see, for example, [5, Theorem 1.27].

Proposition 2.5. If $f$ is a relatively pointwise recurrent non-recurrent map, then $f$ is not equicontinuous.

Proof. Since $f$ is a relatively pointwise recurrent non-recurrent map, then $R(f)$ is not closed and so is not equal to $\Omega(f)$. From [10, Proposition 2.1] it follows that $f$ is not equicontinuous.

The following proposition can be derived from [10, Proposition 2.1].

Proposition 2.6. If $f$ is an equicontinuous relatively pointwise recurrent map, then $f$ is a pointwise recurrent map. 


\section{Proof of MAin theorem}

Before going into the proof of the main theorem we recall the definition of a graph. A (finite) graph $G$ is a compact connected Hausdorf space which contains a finite non-empty set $V$ (the set of vertices), such that every connected component of $G \backslash V$ is homeomorphic to an open interval of the real line. These connected components are called edges. Since any graph can be embedded in $\mathbb{R}^{3}$, in what follows we will consider each graph endowed with the topology induced by the topology of $\mathbb{R}^{3}$. A graph map is a continuous map from a graph $G$ to itself.

To prove the main theorem we need the following lemmas:

Lemma 3.1. Let $f$ be a graph map. Then $\overline{R(f)}=\overline{A P(f)}$.

Proof. We recall that $\overline{A P(f)}$ is the closure of the union of all minimal sets of $f$ and $\overline{A P(f)} \subset \overline{R(f)}$.

In [1, Theorem 1] the authors showed that a minimal set of a graph map is a finite set or a Cantor set or a union of (finitely many) pairwise disjoint circles. One can deduce that each component of $G-(\overline{A P(f)} \cup V)$ is an open $\operatorname{arc}$ of $G$.

Let $] a, b[$ be a component of $G-(\overline{A P(f)} \cup V)$ such that $a \in \overline{A P(f)}$. We suppose that $] a, b[\cap R(f) \neq \emptyset$. Let $x$ be an element of $] a, b[\cap R(f)$. Since $x$ is recurrent, there exists an increasing sequence $\left(n_{q}\right)$ such that $\left(f^{n_{q}}(x)\right)$ converges to $x$. There exist three integers $i<j<k$ such that one of the following two statement holds:

(1) $a<x<f^{n_{k}}(x)<f^{n_{j}}(x)<f^{n_{i}}(x)$.

(2) $a<f^{n_{i}}(x)<f^{n_{j}}(x)<f^{n_{k}}(x)<x$.

By applying [9, Lemma 2.2] we obtain:

(1) $\left.\Rightarrow f^{n_{k}}(a) \in\right] a, b[$.

$\left.(2) \Rightarrow f^{n_{j}-n_{i}}(a) \in\right] a, b[$.

In both cases the interval $] a, b[$ will intersect $\overline{A P(f)}$, which is impossible.

Lemma 3.2. Let $f$ be a relatively pointwise recurrent map of a graph $G$. If $W$ is a proper minimal set of $f$, then $W$ is not a union of (finitely many) pairwise disjoint circles.

Proof. In [1, Theorem 1] the authors showed that a minimal set of a graph map is a finite set or a Cantor set or a union of (finitely many) pairwise disjoint circles.

Suppose that $W$ is a union of (finitely many) pairwise disjoint circles. Then $G$ is not a circle (because $W \neq G$ ). From the fact that $G$ is connected it follows that $W$ contains a branching point $w$. Let $A$ be an arc of $G$ such that $A \cap W=\{w\}$. Since $\overline{R(f)}=G$, there exists in $A$ a sequence $\left(w_{n}\right)$ of recurrent points which converges to $w$. From the fact that $O(w, f)$ is dense in $W$ it follows that there exists an integer $p$ such that $f^{p}(w)$ is not a branching point and so by continuity of $f^{p}$, there exists an integer $N$ such that $f^{p}\left(w_{n}\right) \in W$ for all $n>N$. The recurrence of $w_{n}$ implies that there exists an integer $q>p$ such that $f^{q}\left(w_{n}\right) \in A-\{w\}$, which implies that $f^{q-p}\left(f^{p}\left(w_{n}\right)\right) \in A-\{w\}$, which contradicts the fact that $W$ is invariant.

Lemma 3.3. Let $f$ be a relatively pointwise recurrent map of a graph $G$. If $f$ is not a minimal map, then $\overline{P(f)}=\overline{A P(f)}$.

Proof. We always have the inclusion $\overline{P(f)} \subset \overline{A P(f)}$.

By applying [1, Theorem 1] and Lemma 3.2 it follows that every minimal set of $f$ is a periodic orbit or a Cantor set. Let $W$ be a minimal set which is a 
Cantor set. Let $w$ be an element of $W$ and let $w^{\prime}$ be an element of $G-V(G)$ such that the open arc $\left(w, w^{\prime}\right)$ does not meet $W$. If $\left(w, w^{\prime}\right) \cap P(f)=\emptyset$, then from the fact that $\left(w, w^{\prime}\right) \cap R(f) \neq \emptyset$ and by applying [9, Lemma 2.2] it follows that $\left(w, w^{\prime}\right) \cap O(w, f) \neq \emptyset$, which is impossible. Thus $w \in \overline{P(f)}$. Since $P(f)$ is invariant, $\overline{O(w, f)} \subset \overline{P(f)}$ and so $W \subset \overline{P(f)}$. Therefore $\overline{A P(f)}=\overline{P(f)}$.

Proof of the main theorem. The two statements imply that $f$ is a relatively pointwise recurrent map.

Conversely, (1) if $f$ is a minimal map, then first by [11, Theorem 3.2] it follows that $G$ is a circle, and second by [11, Lemma 3.1] it follows that $f$ is a homeomorphism. From Proposition 2.4 it follows that $f$ is a pointwise non-wandering circle map without periodic points. Thus it is topologically conjugate to an irrational rotation.

(2) If $f$ is not a minimal map, then from Lemma 3.1 and Lemma 3.3 it follows that $\overline{P(f)}=G$.

The fact that the set of periodic points of a transitive graph map is dense was proved by Blokh in [3]. The following corollary extends this result.

Corollary 3.4. Let $f$ be a relatively pointwise recurrent map of a graph $G$. If $f$ is not a minimal map, then $\overline{P(f)}=G$.

\section{ACKNOWLEDGEMENT}

The author gratefully acknowledges the helpful corrections, comments and suggestions from the referee.

\section{REFERENCES}

1. F. Balibrea, R. Hric and L. Snoha, Minimal sets on graphs and dendrites, Internat. J. Bifur. Chaos Appl. Sci. Engrg. 13 (2003), 1721-1725. MR2015622 (2004i:37083)

2. G.D. Birkhoff, Dynamical Systems, revised edition, Amer. Math. Soc. Colloq. Publ., vol. IX, Amer. Math. Soc., Providence, RI, 1966. MR0209095 (35:1)

3. A. Blokh, On the connection between entropy and transitivity for one-dimensional mappings, Russian Math. Surveys 42:5 (1987), 165-166. MR928783 (89g:58117)

4. D.B.A. Epstein, Pointwise periodic homeomorphisms, Proc. London Math. Soc. (3) 42 (1981), 415-460. MR614729 (83e:57011)

5. H. Furstenberg, Recurrence in ergodic theory and combinatorial number theory, M. B. Porter Lectures. Princeton University Press, Princeton, 1981. MR603625 (82j:28010)

6. W.H. Gottschalk, Powers of homeomorphisms with almost periodic properties, Bull. Amer. Math. Soc. 50 (1944), 222-227. MR0009850 (5:213c)

7. B. Hasselblatt and A. Katok, A First Course in Dynamics: With a Panorama of Recent Developments, Cambridge University Press, 2003. MR 1995704 (2004f:37001)

8. A. Illanes, A characterization of dendrites with periodic-recurrent property, Topology Proc. 23 (1998), 221-235. MR.1803250 (2001j:54037)

9. J.H. Mai and S. Shao, $\bar{R}=\bar{P} \cup R$ for graph maps, J. Math. Anal. Appl. 350 (2009), 9-11. MR2476886 (2010a:37082)

10. J.H. Mai, The structure of equicontinuous maps, Trans. Amer. Math. Soc. 355 (2003), 41254136 MR $1990578(2004 \mathrm{e}: 54021)$

11. J.H. Mai, Pointwise-recurrent graph maps, Ergod. Th. Dynam. Sys. 25 (2005), 629-637. MR 2129113 (2005m:37094)

12. D. Montgomery, Pointwise periodic homeomorphisms, Amer. J. Math. 59 (1937), 118-120. MR 1507223 
13. Z. Nitecki, Maps of the interval with closed periodic set, Proc. Amer. Math. Soc. 85 (1982), 451-456. MR656122 (83k:58067)

14. L.G. Oversteegen and E.D. Tymchatyn, Recurrent homeomorphisms on $\mathbf{R}^{\mathbf{2}}$ are periodic, Proc. Amer. Math. Soc. 110 (1990), 1083-1088. MR1037216 (91k:54070)

15. N. Weaver, Pointwise periodic homeomorphisms of continua, Ann. of Math. (2) 95 (1972), 83-85. MR 0293600 (45:2677)

Institut Supérieur d'Informatique et du Multimedia, Route de Tunis, Km 10, B.P. 242, SFAX 3021, TUNISIA

E-mail address: hattab.hawete@yahoo.fr 